\title{
Evaluation of Maize Streak Virus Pathogenicity in Differentially Resistant Zea mays Genotypes
}

\author{
D. P. Martin, J. A. Willment, and E. P. Rybicki
}

Microbiology Department, University of Cape Town, Private Bag, Rondebosch, Western Cape, South Africa 7701. Accepted for publication 7 May 1999.

\begin{abstract}
Martin, D. P., Willment, J. A., and Rybicki, E. P. 1999. Evaluation of maize streak virus pathogenicity in differentially resistant Zea mays genotypes. Phytopathology 89:695-700.

We devised a rapid technique for the objective and precise assessment of both the pathogenicity of maize streak virus (MSV) isolates and the MSV resistance of maize genotypes. The technique involves the use of agroinoculation to infect maize seedlings and the objective symptom evaluation by quantification of infection rates, stunting, and chlorotic leaf areas. In assessing the MSV resistance of 19 maize genotypes, we de-

ysis of MSV resistance phenotypes, ranging from extremely susceptible to completely immune. We further demonstrate how quantification of chlorotic leaf areas by image analysis permits differentiation between degrees of MSV resistance that are indistinguishable from one another using currently employed symptom assessment approaches. Using chlorotic area measurements, we quantify the virulence of a diverse group of $10 \mathrm{MSV}$ isolates and, through agroinoculation of differentially susceptible maize genotypes, we demonstrate the use of our technique in evaluating the pathogenicity of these isolates.
\end{abstract} scribe how the use of differentially virulent virus isolates enables the anal-
Additional keywords: breeding, maize streak disease.
Maize streak virus (MSV), the type member of the genus Mastrevirus of the family Geminiviridae, is the causal agent of maize streak disease, the most significant and widespread maize disease in sub-Saharan Africa (2). Control of the disease through control of its insect vector using carbamate insecticides is effective, but beyond the means of the vast majority of African farmers (4). The belief that the best solution to the African MSV problem is the widespread use of high-yielding MSV-resistant varieties has led to the initiation of maize breeding programs aimed at developing such varieties throughout Africa $(1,4,11,15)$.

A number of techniques for the quantification of MSV symptoms has been devised for breeding purposes and pathology studies. Methods currently employed by breeders are semiquantitative and most commonly involve the use of 5- to 12-point rating systems based on the intensity of streaking $(1,8,11,15)$. While these rating techniques have the advantage of being rapid enough to permit the evaluation of large numbers of infected plants, their reliance on visual estimation of chlorotic areas makes them fundamentally subjective. Besides the visual estimation of chlorotic areas, MSV pathology studies have employed measurements of infection rates (3, 6), chlorophyll concentrations, viral DNA concentrations (3), chlorotic leaf areas $(10,11)$, and stunting (6) to objectively quantify the virulence of MSV isolates.

While natural transmission of MSV is mediated exclusively by a number of leafhopper species in the genus Cicadulina (14), Grimsley et al. (5) developed an alternative means of transmitting MSV using Agrobacterium tumefaciens-mediated transfer of tandemly cloned complete or partial dimers of MSV replicative forms in binary cloning vectors (called agroinfectious constructs). This technique, called agroinfection or agroinoculation, enables the highly efficient and reproducible infection of maize plants with precise concentrations of single MSV isolates.

Corresponding author: E. P. Rybicki; E-mail address: ed@molbiol.uct.ac.za

Publication no. P-1999-0624-01R

(C) 1999 The American Phytopathological Society
Here, we describe a technique for accurately and objectively evaluating both the pathogenicity of MSV isolates and the MSV resistance of maize genotypes. The technique incorporates agroinoculation as a means of transmitting specific virus isolates and a symptom quantification approach that integrates measurements of chlorotic leaf areas, degrees of virus-induced stunting, and infection rates. Besides providing a valuable tool for studying the virulence of MSV isolates, the technique enables the rapid differentiation between the relative MSV resistance of maize genotypes with a degree of resolution and objectivity not attainable with currently employed resistance evaluation techniques.

\section{MATERIALS AND METHODS}

Maize genotypes and virus strains. Seed of the inbred maize lines Z391, Z446, Z459, Z470, Z471, and Z491 was supplied by K. Pixley (International Maize and Wheat Improvement Center [CIMMYT], Harare, Zimbabwe). Seed of the inbred lines P608, P612, CML204, Mo17, and B73 was provided by J. B. J. van Rensburg (Summer Grains Centre [SGC], Potchefstroom, South Africa). Seed of the hybrids PAN6099, PAN6191, PAN6193, PAN6195, PAN6363, PAN6364, and PAN6549 was obtained from D. Nowell (PANNAR Ltd., Greytown, South Africa). Sweet corn (cv. Jubilee) seed was obtained from Starke Ayres Nursery (Cape Town, South Africa). Agroinfectious constructs of MSV isolates (Table 1) were obtained from M. Boulton (pNs; John Innes Centre, Norwich, United Kingdom) or M. Peterschmitt (pR2X2; Centre de Coopération Internationale en Recherche Agronomique pour le Développement [CIRAD], Montpellier, France) or produced in this laboratory (pMatA, pKom, pSet, pVM, pVW, pTas, pMatB, pMtKA, and pWW; D. P. Martin, J. A. Willment, W. Schnippenkoetter, and E. P. Rybicki, unpublished data). Preliminary studies indicated that these MSV isolates produced symptoms in sweet corn ranging from very severe to very mild (D. P. Martin, J. A. Willment, W. Schnippenkoetter, and E. P. Rybicki, unpublished data). A. tumefaciens C58C1 (pMP90) (9) was used to deliver constructs during agroinoculation.

Agroinoculation. Construction of agroinfectious clones was carried out according to Grimsley et al. (5). To permit efficient repli- 
cative release upon agroinfection, all constructs contained one full MSV genome bound by two full, large, intergenic region genomic components (13). Agroinoculation was performed according to Grimsley et al. (5) with the following modifications: (i) standardized inoculum was obtained from actively growing A. tumefaciens cultures with an optical density at $600 \mathrm{~nm}$ of 0.4 and were concentrated 10-fold after two washes with sterile distilled water and stored on ice for no more than $30 \mathrm{~min}$ before injection; (ii) 3-day-old seedlings were injected; (iii) $2 \mu \mathrm{l}$ of inoculum were injected $1 \mathrm{~mm}$ beneath the coleoptilar node of seedlings using a 25- $\mu$ l Hamilton syringe (Hamilton, Bonaduz, Switzerland), with its beveled tip inserted to a standardized depth of $1.5 \mathrm{~mm}$ (aided by a specifically designed collar permitting penetration of only $1.5 \mathrm{~mm}$ of the tip into the coleoptile); and (iv) only seedlings with a coleoptile between 15 and $30 \mathrm{~mm}$ in length were selected for injection. Agroinfection experiments involving specific constructs were carried out on groups of 14 plants. For each agroinfection experiment, a group of 14 seedlings injected with sterile distilled water served as an uninfected control. Seedlings were grown under near-uniform conditions in a plant growth room maintained at 21 to $22^{\circ} \mathrm{C}, 80 \%$ relative humidity, with $16 \mathrm{~h}$ of light per day.

Analysis of symptoms. Disease severity was measured in terms of (i) percentages of agroinoculated plants that became infected, (ii) percentages of leaf areas covered by chlorotic lesions in infected plants, and (iii) heights of infected plants relative to uninfected control plants. The proportion of plants showing symptoms was determined at 3-day intervals between 5 and 14 days after injection. For each MSV isolate-maize genotype combination, these measurements were integrated into an infection rate value $(I R)$ calculated as the mean percentage of infected plants observed at the four assessment times. Percentage of leaf area covered by chlorotic lesions in symptomatic plants was estimated for leaves two through six using a previously described microcomputer-based image analysis technique (10). The image analysis protocol required the use of a digital image capture device and analysis software developed in our laboratory (available online free of charge from the University of Cape Town). The percentage of chlorotic areas of leaves

TABLE 1. Agroinfectious maize streak virus (MSV) constructs

\begin{tabular}{llll}
\hline Construct & MSV isolate & Source of isolate & Country of origin \\
\hline pMatA & MatA & Zea mays & Zimbabwe \\
pKom & Kom & Zea mays & South Africa \\
pVM & VM & Zea mays & South Africa \\
pNs & Ns & Zea mays & Nigeria \\
pR2X2 & R2 & Zea mays & Reunion \\
pMtKA & MtKA & Zea mays & Kenya \\
pMatB & MatB & Zea mays & Zimbabwe \\
pVW & VW & Triticum aestivum & South Africa \\
pTas & Tas & Triticum aestivum & South Africa \\
pWW & WW & Triticum aestivum & South Africa \\
pSet & Set & Setaria sp. & South Africa \\
\hline
\end{tabular}

TABLE 2. Virulence of maize streak virus (MSV) isolates determined through agroinoculation of differentially susceptible maize genotypes

\begin{tabular}{lrrrr}
\hline \multirow{2}{*}{$\begin{array}{l}\text { Agroinfectious } \\
\text { construct }\end{array}$} & \multicolumn{3}{c}{$C_{4-6}{ }^{\mathrm{a}}\left(\mathrm{CI}_{95}{ }^{\mathrm{b}}\right)$} & \\
\cline { 2 - 4 } & \multicolumn{1}{c}{$\mathrm{B} 73$} & Sweet corn & PAN6099 & \multicolumn{1}{c}{$M C_{4-6}\left(\mathrm{CI}_{95}\right)$} \\
\hline pMatA & $83.6(1.6)$ & $79.5(0.6)$ & $61.3(1.7)$ & $74.8(1.1)$ \\
pMatB & $80.8(6.2)$ & $73.0(0.2)$ & $66.9(4.1)$ & $73.6(4.2)$ \\
pNs & $81.9(1.2)$ & $76.3(1.2)$ & $55.9(2.9)$ & $71.4(1.0)$ \\
pR2X2 & $83.2(1.2)$ & $56.0(2.6)$ & $51.5(4.0)$ & $64.1(1.5)$ \\
pMtKA & $82.2(1.2)$ & $64.4(2.7)$ & $43.3(1.8)$ & $63.2(1.5)$ \\
pKom & $76.0(2.8)$ & $55.3(2.3)$ & $32.8(1.8)$ & $55.6(2.1)$ \\
pVW & $78.8(4.0)$ & $55.6(4.3)$ & $28.9(0.8)$ & $54.4(1.5)$ \\
pTas & $65.8(4.0)$ & $31.1(3.0)$ & 0 & $32.3(0.9)$ \\
pSet & $16.9(2.1)$ & $23.4(0.9)$ & 0 & $13.4(1.5)$ \\
pVM & $4.7(1.0)$ & $11.8(1.7)$ & 0 & $5.5(0.9)$ \\
\hline
\end{tabular}

a Average chlorotic area occurring on leaves four through six.

b $95 \%$ confidence interval in parentheses. two and three was assessed 15 days after agroinoculation; the percentage of chlorotic areas of leaves four, five, and six was assessed 22, 29, and 35 days after agroinoculation, respectively. For each MSV isolate-maize genotype combination, the mean percentage of chlorotic area of either the second to sixth leaves (designated $C_{2-6}$ ) or the fourth to sixth leaves (designated $C_{4-6}$ ) was used as a representative measure of chlorosis. Heights of symptomatic plants and uninfected control plants were measured 15 days after agroinoculation as the distance from their coleoptilar nodes to the tip of their fourth leaves. For specific MSV isolate-maize genotype combinations, a value (designated $S$; representing stunting occurring as a result of infection) was calculated as the mean height of symptomatic plants expressed as a proportion of the mean height of uninfected control plants. The value of $1-S$ was used as a description of stunting. Resistance was rated on a commonly used six-point scale $(0=$ immune to $5=$ highly susceptible $)$ for each of the maize genotypes by the breeders that provided them for use in this study. A resistance rating for sweet corn was supplied by SGC.

Evaluation of MSV strains and maize genotypes. Each MSV isolate was used to infect three maize genotypes: B73 (very susceptible), sweet corn (moderately susceptible), and PAN6099 (moderately resistant). Agroinfection experiments involving each MSV isolate-maize genotype combination were repeated at least three times. For each MSV isolate, a single value (designated $M C_{4-6}$; representing the mean of the $C_{4-6}$ values calculated for B73, sweet corn, and PAN6099) was used as a generalized measure of virulence. Of the MSV isolates tested, severe (MSV-MatA), moderately severe (MSV-Kom), and mild (MSV-Set) isolates were selected for the evaluation of MSV resistance in 19 maize genotypes. The MSVMatA, MSV-Kom, and MSV-Set agroinfectious constructs used were pMatA, pKom, and pSet, respectively. Each maize line was challenged with these three isolates in at least three agroinfection experiments. For each agroinfection experiment involving a specific maize genotype, the values $M I R, M 1-S$, and $M C_{2-6}$ (representing the mean $I R, 1-S$, and $C_{2-6}$ values, respectively, determined for the three test MSV isolates) were used as generalized measures of resistance.

\section{RESULTS}

Chlorotic lesions on the second or third leaves of agroinfected plants first appeared between 4 and 11 days after injection. With very few exceptions, plants that became infected showed symp-

TABLE 3. Averaged maize streak virus (MSV) symptoms in maize genotypes infected with isolates MSV-MatA, MSV-Kom, and MSV-Set

\begin{tabular}{lcccc}
\hline Genotype & $M I R^{\mathrm{a}}\left(\mathrm{CI}_{95}{ }^{\mathrm{b}}\right)$ & $M 1-S^{\mathrm{c}}\left(\mathrm{CI}_{95}\right)$ & $M C_{2-6}{ }^{\mathrm{d}}\left(\mathrm{CI}_{95}\right)$ & Rating \\
\hline B73 & $82.2(2.4)$ & $0.351(0.038)$ & $50.4(0.7)$ & 5 \\
Sweet corn & $81.6(4.8)$ & $0.262(0.017)$ & $45.7(1.5)$ & 5 \\
M017 & $41.0(11.7)$ & $0.289(0.087)$ & $35.4(3.2)$ & 5 \\
PAN6363 & $51.1(5.0)$ & $0.162(0.017)$ & $31.7(1.5)$ & 5 \\
PAN6364 & $46.6(3.2)$ & $0.138(0.009)$ & $30.0(1.4)$ & 5 \\
PAN6549 & $49.9(4.6)$ & $0.110(0.068)$ & $30.6(0.7)$ & 5 \\
PAN6193 & $54.5(4.5)$ & $0.177(0.006)$ & $22.7(2.8)$ & 2 \\
PAN6191 & $45.8(3.8)$ & $0.163(0.021)$ & $22.7(2.0)$ & 2 \\
PAN6195 & $45.1(1.6)$ & $0.127(0.011)$ & $25.3(1.6)$ & 2 \\
PAN6099 & $41.9(2.6)$ & $0.121(0.011)$ & $23.3(1.3)$ & 1 \\
Z459 & $59.4(3.6)$ & $0.116(0.056)$ & $26.0(0.9)$ & 0 \\
P612 & $38.7(10.4)$ & $0.086(0.051)$ & $28.2(0.3)$ & 0 \\
Z391 & $48.1(1.9)$ & $0.110(0.056)$ & $15.1(1.3)$ & 0 \\
CML204 & $28.0(6.7)$ & $0.140(0.052)$ & $15.8(3.6)$ & 0 \\
P608 & $25.4(10.9)$ & $0.067(0.012)$ & $19.2(1.4)$ & 0 \\
Z446 & $50.9(1.8)$ & $0.049(0.007)$ & $12.7(2.0)$ & 0 \\
Z471 & $21.6(5.0)$ & $0.022(0.023)$ & $8.1(3.8)$ & 0 \\
Z470 & $4.7(5.1)$ & $0.049(0.047)$ & $1.3(0.8)$ & 0 \\
Z491 & $7.6(6.8)$ & $0.010(0.035)$ & $2.1(1.6)$ & 0 \\
-
\end{tabular}

a Average infection rate.

b $95 \%$ confidence interval in parentheses.

c Average degree of stunting.

d Average chlorotic area occurring on leaves two through six. 
toms by 11 days after agroinoculation. Neither chlorotic streaking nor stunting were observed in plants injected with distilled water, A. tumefaciens $\mathrm{C} 58 \mathrm{C} 1$ (pMP90) containing pWW (a nonagroinfectious construct of the MSV isolate MSV-WW), or A. tumefaciens $\mathrm{C} 58 \mathrm{C} 1$ (pMP90) containing the binary cloning vector $\mathrm{pBI} 121$ without an MSV genome.

Although certain aspects of agroinfectious plasmid construction can influence the performance of constructs during the early stages of infection, we observed that chlorotic areas occurring on the fourth and subsequent leaves of successfully infected plants are not subject to these influences (data not shown). Because the agroinfectious plasmids used in this study had been constructed using various cloning vectors and cloning strategies, the virulences of agroinfectious MSV isolates were compared with one another using only their $C_{4-6}$ values.

A wide range of $C_{4-6}$ values was observed for the $10 \mathrm{MSV}$ isolates used to agroinfect B73, sweet corn, and PAN6099 (Table 2). By testing the virulence of the isolates in differentially susceptible maize lines, it was possible to differentiate between them by the symptoms they caused. The relative virulences of the mild isolates MSV-Tas, MSV-Set, and MSV-VW could be most effectively analyzed in B73, an extremely susceptible inbred line. While B73 and sweet corn were susceptible to all the isolates tested, PAN6099 was completely immune to the mild isolates. In sweet corn (a moderately susceptible variety) and PAN6099 (a moderately resistant hybrid), clear differentiation between infection symptoms of the more severe MSV isolates was achieved. Based on the $C_{4-6}$ values obtained for the more virulent isolates in sweet corn, these isolates could be subdivided into severe and moderately severe groups. While the severe group had $C_{2-6}$ values between 73.0 and 79.5 and consisted of MSV-MatA, MSV-MatB, and MSV-Ns, the moderately severe group with $C_{4-6}$ values between 55.3 and 64.4 comprised MSV-Kom, MSV-VM, MSV-MtKA, and MSV-R2. In PAN6099, distinction between these two groups was less defined, with MSV-R2 appearing significantly more virulent than the other moderately severe isolates and MSV-Ns appearing significantly less virulent than the other severe isolates.

The relative severities of the isolates were different when infecting sweet corn and PAN6099. In sweet corn, MSV-MatA was more severe than MSV-MatB, but in PAN6099, MSV-MatB was more severe than MSV-MatA. Similarly, while MSV-R2 was less severe in sweet corn than MSV-MtKA, it was more severe than MSV-MtKA in PAN6099.

We selected MSV-MatA, MSV-Kom, and MSV-Set (representative of the entire range of MSV virulences observed in B73, sweet corn, and PAN6099) for testing the MSV resistance of maize genotypes.

Assessing the susceptibility of maize genotypes to MSV. Based on measurements of mean infection rates $(M I R)$, stunting $(M 1-S)$, and chlorotic areas $\left(M C_{2-6}\right)$, the three most resistant maize lines examined were Z491, Z470, and Z471 (Table 3). With the exception of Z459 and P612, ranking of the lines in order of MSV resistance using our technique corresponded well with rankings based on MSV resistance ratings made by the breeders who produced the lines (Table 3, Fig. 1). While P612 and Z459 were rated

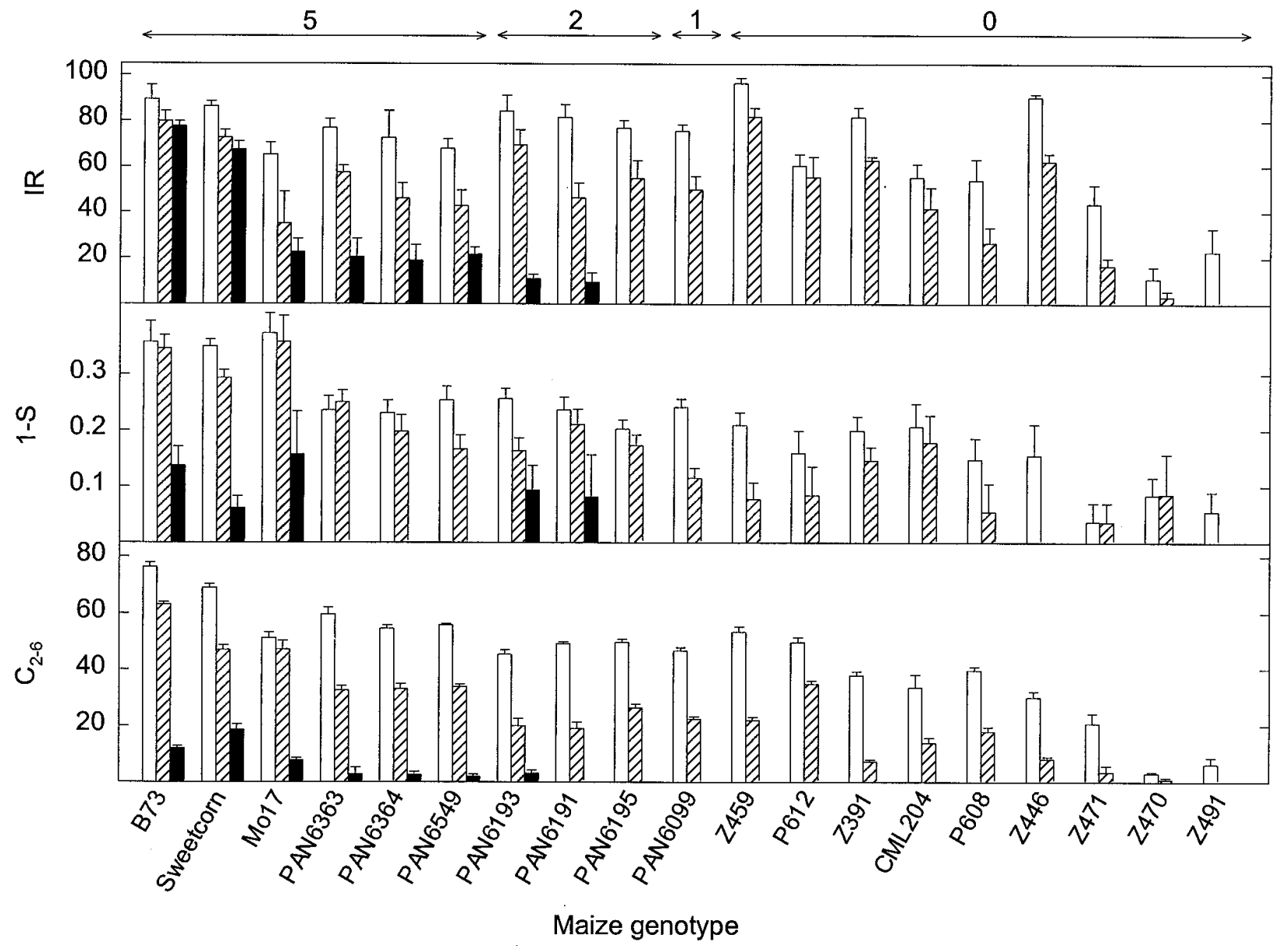

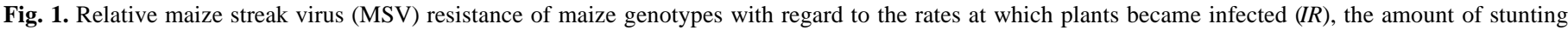

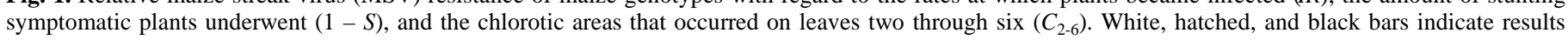

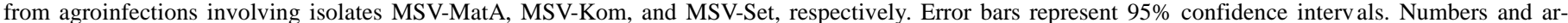

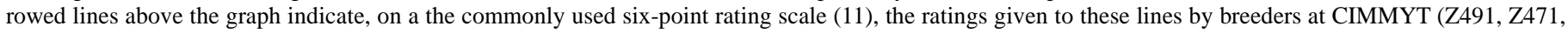

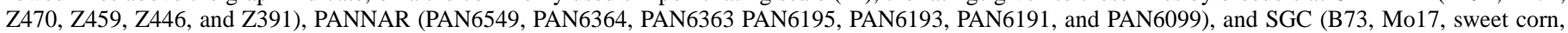
P612, P608, and CML204). 
as entirely immune by the breeders at SGC and CIMMYT, respectively, the $C_{2-6}$ values of these lines when infected with MSV-MatA and MSV-Kom were similar to those of the susceptible PANNAR hybrids (PAN6364, PAN6363, and PAN6549) (Fig. 1). We noted, however, that P612 and Z459 had definite resistance characteristics. They were both immune to MSV-Set and, particularly in the case of P612, were significantly less prone to MSV-induced stunting than were the susceptible PANNAR hybrids. Additionally, mean chlorotic areas on individual leaves of MSV-MatA- and MSV-Kom-infected P612 indicated that, while percentages of chlorotic areas were greatest on leaf five $\left(78.5 \pm 6.1 \% 95 \%\right.$ confidence interval $\left[\mathrm{CI}_{95}\right]$ for MSV-MatA and $67.5 \pm 4.3 \% \mathrm{CI}_{95}$ for MSV-Kom), they had significantly declined by leaf six $\left(58.4 \pm 3.4 \% \mathrm{CI}_{95}\right.$ for MSV-MatA and $24.8 \pm 4.3 \% \mathrm{CI}_{95}$ for MSV-Kom).

Our analysis of symptoms revealed significant differences in MSV resistance within the groups of lines rated by breeders as either completely immune (score of 0 ) or completely susceptible (score of 5). Lines rated as immune using resistance screening methods at CIMMYT (Z391, Z446, Z459, Z470, Z471, and Z491) and SGC (CML204, P612, and P608) were all susceptible to agroinfection with both MSV-MatA and, with the exception of Z491, MSV-Kom. Differentiation between disease symptoms that occurred in all these "immune" lines was possible using our technique (Table 3). The most striking differences between these lines were observed by analysis of chlorotic areas following infection with MSV-MatA (Fig. 2). Similarly, chlorotic area measurements in MSV-Kom-infected lines that had been rated as uniformly susceptible using current assessment approaches varied significantly in their relative susceptibilities to this isolate (Fig. 2).

\section{DISCUSSION}

Precise information on the symptomatic responses of plants to their pathogens is extremely useful both for studying the virulence of different pathogen strains and for breeding plants with elevated pathogen resistance. Although breeding for MSV-resistant maize has yielded germ plasm with significantly enhanced resistance (1, $12,15)$, current procedures for testing the resistance of maize lines do not have sufficient resolution to differentiate between lines with similar resistance and are not exacting enough to properly examine highly resistant lines. Also, these resistance screening procedures yield information only on the resistance of maize lines to specific MSV strains; information that is not necessarily accurate or relevant when the lines are challenged with different strains (12). Here, we describe a technique we devised that addresses these problems and is applicable to the analysis of both the virulence of MSV isolates and the MSV resistance of maize genotypes.

Use of susceptible, moderately susceptible, and moderately resistant maize genotypes for evaluating the virulence of MSV isolates enabled reliable differentiation of all the isolates investigated. The milder isolates (MSV-VW, MSV-Set, and MSV-Tas; with $C_{4-6}$ values between 5.5 and 32.3) are genetically very distinct from the more severe isolates (with $C_{4-6}$ values between 54.5 and 74.8) (Fig. 3 ). Based on the analysis of full genomic sequences, MSV-Set shares an average of only $78 \%$ sequence identity with the isolates obtained from maize, while MSV-VW and MSV-Tas each share $89 \%$ sequence identity with the isolates obtained from maize (J. A. Willment, W. Schnippenkoetter, and E. P. Rybicki, unpublished data). This probably indicates that MSV-VM, MSV-Set, and MSV-Tas are specifically adapted to infection of grass species other than maize. Within the group of isolates that produce severe symptoms in maize, there are further three distinct groupings (Fig. 3). The first group, representing viruses from Zimbabwe (MSV-MatA and MSV-MatB), Kenya (MSV-MtKA), and Nigeria (MSV-Ns), produced the severest symptoms on both sweet corn and, with the exception of MSV-MtKA, PAN6099. The second and third groups containing viruses from South Africa (MSV-Kom and MSV-VM) and Reunion (MSV-R2) were moderately severe in sweet corn, while MSV-R2 was significantly more severe than the South African isolates in PAN6099. The variation in relative virulences of the MSV isolates examined in this study when infecting sweet corn and PAN6099 indicates that, amongst maize genotypes, virus isolates apparently display a degree of host adaptation.

Inheritance of MSV resistance in maize is complex, involving at least three major genes and an undetermined number of minor genes $(8,12,15,16)$. Because of its sensitivity, our technique is ideally suited to analyzing the continuum of resistance phenotypes that one would expect with quantitatively inherited resistance. We were capable of resolving differences between the resistance of genotypes rated uniformly immune by breeders, indicating that the genotypes we tested possibly contained a highly heterogeneous mix of MSV resistance genes. Using our technique, it was also possible to detect
A

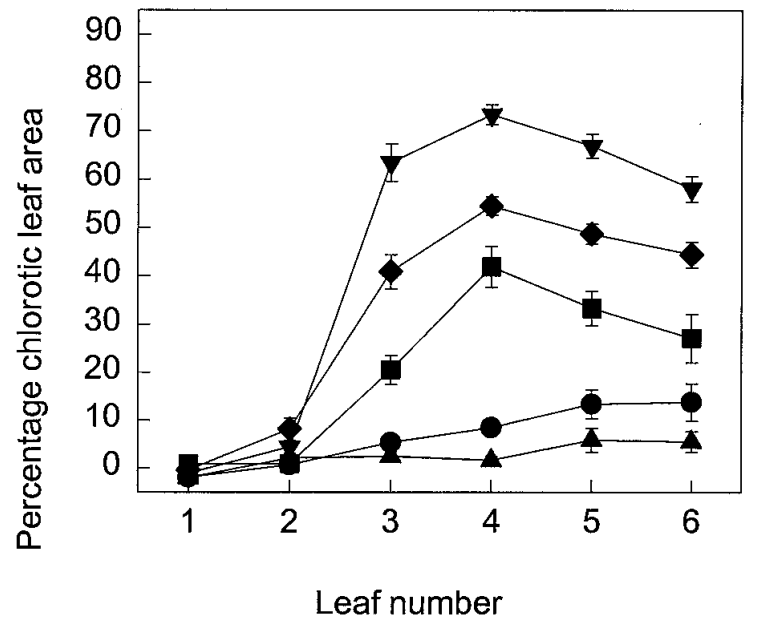

B

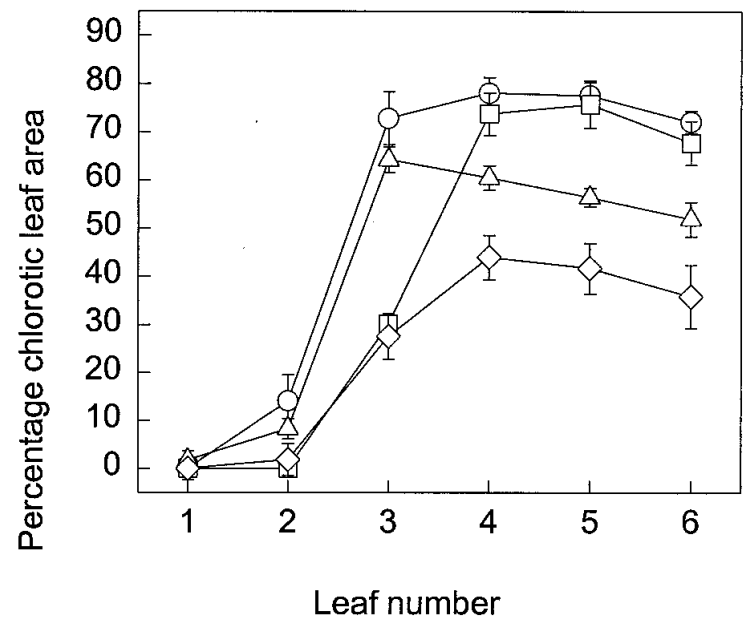

Fig. 2. Use of percentage of chlorotic area measurements on the first six leaves of agroinfected plants to determine the relative resistance of maize genotypes with degrees of maize streak virus (MSV) resistance that are not readily distinguishable using currently employed resistance screening approaches. A, Lines

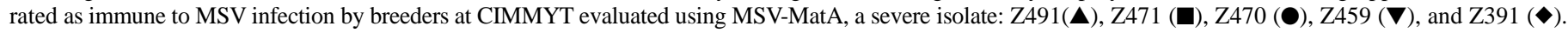
B, Genotypes rated as having no resistance to MSV by breeders at SGC (B73 [O], Mo17 [ $\square$ ], and sweet corn $[\triangle$ ]) and PANNAR (PAN6364 [ $\diamond])$ evaluated using MSV-Kom, a moderately severe isolate. Error bars represent $95 \%$ confidence intervals. 
differences in the MSV susceptibilities of genotypes rated as completely susceptible by breeders, indicating that a number of potentially useful minor resistance genes may exist undetected in genotypes previously considered to have no MSV resistance.

With two exceptions, our resistance assessments were essentially in accordance with those of the breeders that had produced the genotypes used in this study. While lines P612 and Z459 were rated entirely immune by breeders at SGC and CIMMYT, respectively, they displayed streak symptoms characteristic of susceptible genotypes when agroinoculated with MSV-MatA and MSV-Kom. These lines were possibly (i) resistant to infection at a later developmental stage, (ii) resistant to different MSV strains to those we used, (iii) resistant to MSV infection via the leafhopper transmission route, or (iv) not resistant to MSV but either completely resistant to leafhopper feeding or less palatable to leafhoppers than other lines being tested at the same time at CIMMYT or SGC. This latter point is highly relevant since, at SGC and CIMMYT, different lines are tested beside one another in rows and in neither case are viruliferous leafhoppers restricted to feeding on specific plants. While SGC relies on natural infection (15), CIMMYT relies on the use of anaesthetized leafhoppers that feed on plants onto which they are placed $(2,16)$. PANNAR overcomes problems with leafhopper feeding preferences by placing viruliferous leafhoppers in small cages attached to the second leaf of plants being tested for resistance (1). Regardless of the reasons that P612 and Z459 appeared immune to MSV during testing at SGC and CIMMYT, respectively, the lines do appear to have a measure of MSV resistance in that they are immune to MSV-Set and experience less-severe stunting than the susceptible genotypes. Differences in the resistance traits of P612 and Z459 from the other resistant genotypes examined in this study highlight the fact that various mechanisms for MSV resistance exist.

In this study, we employed mild, moderate, and severe agroinfectious MSV isolates for screening maize genotypes in order to

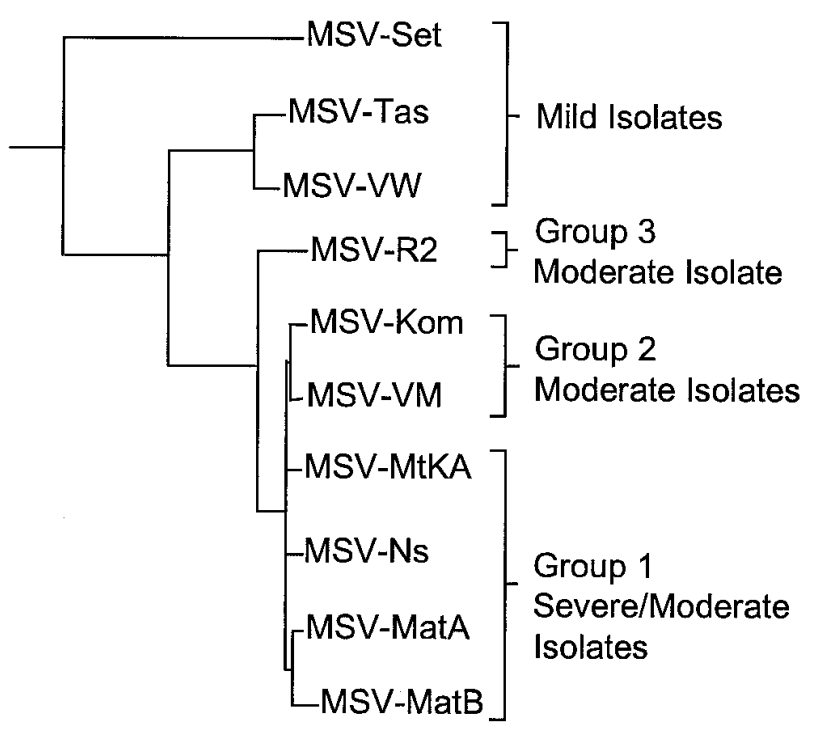

Fig. 3. Phylogenetic tree showing the relationship between the maize streak virus (MSV) isolates examined in this study. Construction of the tree was carried out using DNAMAN (version 3; Lynnon BioSoft, Quebec, Canada) based on sequences of a 1,300-base pair fragment of these viruses' genomes ranging from 1,746 to 288 (with nucleotides one and two the A and C nucleotides, respectively, of the conserved geminivirus TAATATTAC stem-loop sequence). Sequences for this region were obtained from the GenBank accession numbers AJ224504 (MSV-R2), X01633 (MSV-Ns), AJ012633 (MSV-MatA), AJ012634 (MSV-MatB), AJ012640 (MSV-MtKA), AF003952 (MSV-Kom), AF007881 (MSV-Set), AJ012638 (MSV-VW), AJ012637 (MSV-VM), and AJ012636 (MSV-Tas). Horizontal distances are proportional to distances; vertical distances are arbitrary. All nodes supported by less than $60 \%$ of a 1,000-repeat bootstrap iteration are collapsed. The tree was rooted on the Panicum streak mastrevirus sequence (data not shown). demonstrate the utility of our technique for the analysis of the broadest possible range of MSV infection severities. For other applications, selection of the strains used would depend on the task at hand. Thus, while the analysis of MSV infection responses in highly resistant maize genotypes would warrant the use of a panel of genetically diverse but highly virulent MSV isolates, analysis of marginally resistant maize genotypes for low levels of polygenically inherited resistance would be best achieved with the use of mild isolates.

Using agroinoculation in our technique as a means of transmitting viruses has several advantages over leafhopper transmission. (i) Not only does agroinfection of 3-day-old seedlings allow virus transmission rates as high as leafhopper transmission of severe MSV isolates, but we also found that it yields higher transmission rates for milder isolates such as MSV-Set and MSV-VW (13). (ii) Agroinoculation allows infection of plants at very specific timepoints with a precise amount of inoculum and avoids the issue of differential feeding preferences of leafhoppers. (iii) Agroinoculation avoids logistical problems associated with mass rearing of viruliferous leafhoppers for simultaneous controlled transmission of different virus isolates. The main limitation of agroinoculation, however, is that $A$. tumefaciens transformed with an agroinfectious MSV construct constitutes a genetically modified organism that cannot be freely released into the environment. This inconvenience is largely offset by the fact that, in an adequate containment facility, there are no constraints on the severity of MSV isolates that can be used for testing maize genotypes. Highly virulent isolates could be obtained either from naturally infected maize in regions known to have severe MSV strains (such as the region in Zimbabwe from which MSV-MatA and MSV-MatB originated) or by purposefully evolving resistance-breaking isolates through controlled laboratory selection (7).

Because of the objectivity afforded by our technique, it could be put to use in standardized schemes for the quantification of both the MSV resistance of maize lines and the virulence of MSV isolates. While it would not be convenient to use the technique for assessing the large numbers of plants commonly examined in large resistance screening programs, we propose its use in a standard supplementary screening protocol for the analysis of promising maize genotypes. Besides a standard means of assessing resistance to MSV isolates, the virulence of MSV isolates determined using a standard assessment protocol would also be of use to maize breeders who would have at their disposal virulence profiles of MSV isolates found in particular locations. With this information, panels of MSV isolates specifically suited to particular resistance screening projects could be conveniently chosen. Plant pathologists studying the epidemiology of MSV would also find data of this nature useful. If resistance and virulence estimates were expressed relative to one or a few standard maize lines or virus isolates, respectively, it should be possible to compare directly the results obtained by different research groups that use our technique.

Relative to techniques in current use, our approach provides greatly enhanced resolution and objectivity in both the comparative assessment of MSV resistance amongst maize genotypes and the comparison of virulence amongst MSV isolates. For the purpose of identifying MSV pathogenicity determinants, we are currently exploiting its sensitivity in analyzing subtle differences in the virulence of laboratory-constructed mutants and recombinant MSV isolates. With regard to the analysis of MSV symptoms for breeding purposes, we believe that our technique could prove invaluable for the analysis of both marginal and extreme degrees of MSV resistance in interesting lines obtained using conventional screening approaches.

\section{ACKNOWLEDGMENTS}

We thank the Foundation for Research Development for funding; J. B. J. van Rensburg, J. S. Passmore, and E. van der Walt for proofreading and 
valuable comments; J. B. J. van Rensburg, D. Nowell, and K. Pixley for providing seed; and M. Boulton and M. Peterschmitt for providing virus clones.

\section{LITERATURE CITED}

1. Barrow, M. R. 1992. Development of maize hybrids resistant to maize streak virus. Crop Prot. 11:267-271.

2. Bosque-Perez, N. A., and Allem, M. S. 1992. Mass rearing of Cicadulina leafhoppers to screen for maize streak virus resistance. A manual. International Institute of Tropical Agriculture, Ibadan, Nigeria.

3. Boulton, M. I., King, D. I., Markham, P. G., Pinner, M. S., and Davies, J. W. 1991. Host range and symptoms are determined by specific domains of the maize streak virus genome. Virology 181:312-318.

4. Efron, Y., Kim, S. K., Fajemisin, J. M., Mareck, J. H., Tang, C. Y., Dabrowski, Z. T., Rossel, H. W., Thottappilly, G., and Buddenhagen, I. W. 1989. Breeding for resistance to maize streak virus: A multidisciplinary team approach. Plant Breed. 103:1-36.

5. Grimsley, N., Hohn, T., Davies, J. W., and Hohn, B. 1987. Agrobacterium-mediated delivery of infectious maize streak virus in maize plants. Nature 325:177-179.

6. Isnard, M., Granter, M., Frutos, R., Reynaud, B., and Peterschmitt, M. 1997. The quasi-species nature of the maize streak virus DNA. Page 19 in: Maize Streak Disease Symposium. South African Agricultural Research Council, Pretoria, South Africa.

7. Isnard, M., Granter, M., Frutos, R., Reynaud, B., and Peterschmitt, M. 1998. Quasi-species nature of the maize streak virus isolates obtained from a population used to assess maize cultivar response to infection. J. Gen. Virol. 79:3091-3099.

8. Kim, S.-K., Efron, Y., Fajemisin, J. M., and Buddenhagen, I. W. 1989. Mode of gene action for resistance in maize to maize streak virus. Crop Sci. 29:890-894

9. Koncz, C., and Schell, J. 1986. The promoter of $T_{L}-$ DNA gene 5 controls the tissue-specific expression of chimaeric genes carried by a novel type of Agrobacterium binary vector. Mol. Gen. Genet. 204:383-396.

10. Martin, D. P., and Rybicki, E. P. 1998. Microcomputer-based quantification of maize streak virus symptoms in Zea mays. Phytopathology 88:422-427.

11. Rodier, A. 1995. Déterminisme génétique de la résistance du maïs (Zea mays L.) au maize streak virus (MSV). Thèse de Doctorat. l'Ecole Nationale Supérieure Agronomique, Rennes, France.

12. Rodier, A., Assié, J., Marchand, J.-L., and Hervé, Y. 1995. Breeding maize lines for complete and partial resistance to maize streak virus (MSV). Euphytica 81:57-70.

13. Schnippenkoetter, W. H. 1998. The use of agroinfectious clones to investigate recombination between distinct maize streak virus strains. $\mathrm{Ph} . \mathrm{D}$. thesis. University of Cape Town, Western Cape, South Africa.

14. Storey, H. H. 1928. Transmission of maize streak disease. Ann. Appl. Biol. 15:1-25.

15. Van Rensburg, G. D. J., Giliomee, J. H., and Pringle, K. L. 1991. Resistance of South African maize hybrids to maize streak virus. S. Afr. J. Plant Soil 8:38-42.

16. Welz, H. G., Schechert, A., Pernet, A., Pixley, K. V., and Geiger, H. H. 1998. A gene for resistance to the maize streak virus in the African CIMMYT maize inbred line CML202. Mol. Breed. 4:147-154. 